\title{
Prostate brachytherapy in Ghana: our initial experience
}

\author{
James Edward Mensah, MBChB, FWACSl, Joel Yarney, MBChB, FC Rad Onc², Verna Vanderpuye, MBChB, FWACS², \\ Evans Akpakli, MBChB, FWACS 3 , Samuel Tagoe, BSc. MSc ${ }^{2}$, Evans Sasu, BSc. MSc² \\ 'Department of Surgery, School of Medicine and Dentistry, College of Health Sciences, University of Ghana Medical School, ${ }^{2}$ National Center \\ for Radiotherapy and Nuclear Medicine, Korle Bu Teaching Hospital, ${ }^{3}$ Department of Surgery, Korle Bu Teaching Hospital, Accra, Ghana
}

\begin{abstract}
Purpose: This study presents the experience of a brachytherapy team in Ghana with a focus on technology transfer and outcome. The team was initially proctored by experienced physicians from Europe and South Africa.

Material and methods: A total of 90 consecutive patients underwent either brachytherapy alone or brachytherapy in combination with external beam radiotherapy for prostate carcinoma between July 2008 and February 2014 at Korle Bu Teaching Hospital, Accra, Ghana. Patients were classified as low-risk, intermediate, and high-risk according to the National Comprehensive Cancer Network (NCCN) criteria. All low-risk and some intermediate risk group patients were treated with seed implantation alone. Some intermediate and all high-risk group patients received brachytherapy combined with external beam radiotherapy.

Results: The median patient age was 64.0 years (range 46-78 years). The median follow-up was 58 months (range 18-74 months). Twelve patients experienced biochemical failure including one patient who had evidence of metastatic disease and died of prostate cancer. Freedom from biochemical failure rates for low, intermediate, and high-risk cases were $95.4 \%, 90.9 \%$, and $70.8 \%$, respectively. Clinical parameters predictive of biochemical outcome included: clinical stage, Gleason score, and risk group. Pre-treatment prostate specific antigen (PSA) was not a statistically significant predictor of biochemical failure. Sixty-nine patients (76.6\%) experienced grade 1 urinary symptoms in the form of frequency, urgency, and poor stream. These symptoms were mostly self-limiting. Four patients needed catheterization for urinary retention (grade 2). One patient developed a recto urethral fistula (grade 3 ) following banding for hemorrhoids.

Conclusions: Our results compare favorably with those reported by other institutions with more extensive experience. We believe therefore that, interstitial permanent brachytherapy can be safely and effectively performed in a resource challenged environment if adequate training and proctoring is provided.
\end{abstract}

J Contemp Brachytherapy 2016; 8, 5: 379-385 DOI: $10.5114 /$ jcb.2016.62972

Key words: brachytherapy, Ghana, LDR, proctoring, prostate cancer, seeds.

\section{Purpose}

Current management options for localized prostate cancer include surgery, radical prostatectomy (RP), radiotherapy, and active surveillance. Radiotherapy is administered as either external-beam radiotherapy (EBRT), interstitial brachytherapy, or a combination of both. Interstitial brachytherapy with permanent seed implantation for localized prostate cancer has rapidly gained popularity over the past decade in USA, Europe, and Asia [1]. Many reports with long term follow-ups are now available, which confirm good outcomes in selected patients with prostate specific antigen (PSA), relapse-free survival rates equivalent to those achieved by surgery [2,3]. However, the excellent results achieved with both low and high dose rate brachytherapy mostly come from single institutions in developed countries with significant experi- ence in prostate brachytherapy [4,5]. Setting up of a prostate brachytherapy service requires expensive equipment [6] and well trained personnel; therefore not surprisingly there have been relatively few reports from Africa [7].

The objective of this study is to report how capacity was built in a local brachytherapy team in Ghana through international collaboration as well as the clinical outcome and complications identified in the first 90 patients treated with a median follow-up of 58 months.

\section{Material and methods}

Prostate specific antigen tests and trans-rectal ultrasound guided biopsy became widely available in Ghana in 2000. This resulted in a rise in the number of patients being diagnosed with organ-confined disease. Facilities for staging including radioisotope bone scan, computed
Address for correspondence: James Edward Mensah, Department of Surgery, School of Medicine and Dentistry, College of Health Sciences, University of Ghana Medical School, P O Box 4236, Accra, Ghana, phone: +233 208179911, e-mail: jemensah@hotmail.com
Received: 04.06.2016

Accepted: 05.09.2016

Published: 31.10.2016 
tomography (CT) scan, and magnetic resonance imaging (MRI) also became locally available. Some patients travelled to South Africa and Europe because there was no prostate brachytherapy facility in West Africa. As at 2008, South Africa was the only country in Africa that had well established prostate brachytherapy centers. We contacted the management of a brachytherapy company with a branch in South Africa with our prostate cancer data, which indicated a potential market for brachytherapy in Ghana. A memorandum of understanding was signed between the company and our hospital. The company provided equipment and training (proctoring by an experienced specialist), whilst the Hospital committed itself to procuring the radioactive seeds.

The first session of brachytherapy included proctoring by a urologist, physicist, and a computer specialist from the planning software provider. A product specialist nurse also accompanied the team to train the nursing and other theatre staff. This onsite proctoring arrangement continued for 2 years. Following adequate transfer of skills, our proctors stayed at home in their respective countries but were available for consultation online or by telephone in case we encountered any technical challenges. Between June 2008 and February 2014, 90 patients with clinically localized prostate cancer underwent trans-perineal interstitial low dose rate permanent prostate brachytherapy with ${ }^{125} \mathrm{I}$ seeds at Korle $\mathrm{Bu}$ Teaching Hospital. All patients were selected for this procedure based on stage and prognostic criteria and International Prostate Symptom Score after consultation with radiations oncologist and urologists. All patients were staged according to the American Joint Committee on Cancer $6^{\text {th }}$ edition clinical staging guidelines [8] using a directed history, physical examination including digital rectal examination, bone scintigraphy following PSA results, and histopathology information. Where indicated, CT and/or MRI was obtained to evaluate the local extent of disease and nodal status. Patients were classified into low-risk group (T1-2a, PSA $<10.0 \mathrm{ng} / \mathrm{ml}$, and Gleason score $\leq 6)$, intermediate-risk group (T2b-c or PSA of $10-20 \mathrm{ng} / \mathrm{ml}$, or Gleason score 7), and high-risk group (T3a or PSA $>20 \mathrm{ng} / \mathrm{ml}$, or Gleason score 8-10) according to the National Comprehensive Cancer Network (NCCN) criteria [9]. Patients with multiple intermediate-risk adverse factors were included in the high-risk group. Patients in the low-risk group were treated with seed implantation alone. All high-risk group patients were treated

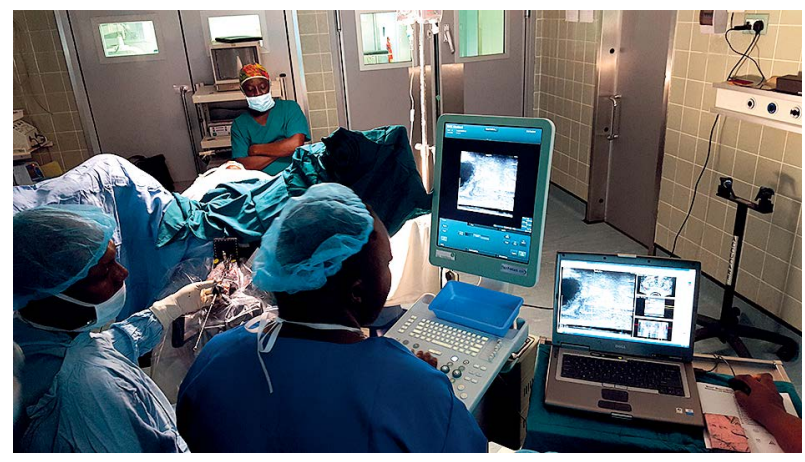

Fig. 1. Real time brachytherapy session with brachytherapy boost combined with EBRT. In the intermediate-risk group, patients with PSA of $15 \mathrm{ng} / \mathrm{ml}$ or less, T1c-2a, Gleason score $3+3$, and percent of biopsy positive cores of less than $50 \%$ were treated by seed implantation alone. Patients with prostate size more than $60 \mathrm{cc}$ received androgen deprivation therapy (ADT) for 3-4 months to reduce prostate size prior to implantation. The ADT comprised luteinizing hormone releasing hormone (LHRH) agonist alone or in combination with an antiandrogen. High risk patients received 18-24 months of ADT. The local ethical committee approval was granted prior to the commencement of the brachytherapy program.

\section{Implant technique and prescription}

Radioiodine seeds were pre-ordered for each patient based on the size of the prostate and whether the implant was radical or boost. Patients were cleared for anesthesia and underwent either general or spinal anesthesia. The real time prostate seed implant technique [10] was used with a perineal template to guide needles into the prostate (Figure 1). A urethral catheter was placed as well as a rectal probe connected to the ultrasound. The prostate volume was confirmed by real time ultrasonography following target delineation. The recommended prescribed dose was 160 Gy for a full implant, and 110 Gy for the boost. The planning system was used to obtain the best isodose coverage with acceptable, urethral, and rectal doses. Assessment of a good dosimetry included the dose to $90 \%$ of the prostate $\left(D_{90}\right)$, the prostate volume receiving $100 \%$ and $150 \%$ of the prescribed dose $\left(V_{100}\right.$ and $V_{150}$ respectively. Upon approval by all members of the team, needles were placed within the prostate and loaded with seeds based on the approved plan. Adjustments during the procedure were based on real-time changes in seed positions and isodose coverage to optimize dosimetry. The patient was discharged home within 24-48 hours after voiding without a catheter. All patients received alpha-blockers just after seed implantation to relieve urinary symptoms for 3-6 months.

\section{External beam radiotherapy protocol}

Patients who received partial implant received additional pelvic irradiation starting 2 months after implant date. These patients are at high risk of extra-prostatic extension and developing lymph node metastases. Doses delivered are between 39.6-50.4 Gy in 20-28 fractions over 4 to 6 weeks using 3-dimensional conformal radiotherapy planning technique on ${ }^{60} \mathrm{Co}$ EBRT machine. The treated volume included the prostate gland, seminal vesicles, periprostatic tissues, and regional lymph nodes with a 0.8 to $1.0 \mathrm{~cm}$ margin, except posteriorly where the margin is $0.5 \mathrm{~cm}$. The choice of prescribed dose depended on $\mathrm{D}_{80}, \mathrm{D}_{100}, \mathrm{D}_{90}$ to the prostate, and doses received by the rectum, urethra, and seminal vesicle with brachytherapy.

\section{Follow-up}

A follow-up was scheduled for two weeks to address any symptoms. The quality of the implant is assessed 
using a CT scan performed a month after the procedure. Patients requiring subsequent external beam irradiation were planned to initiate treatments 12 weeks after seed implantation. Subsequently, patients are monitored by symptom assessment, PSA test, and physical examination every 3-6 months for the first 2 years and every 6 months thereafter. PSA control was determined with respect to freedom from biochemical failure $(\mathrm{FBF})$, which was defined using the nadir $+2 \mathrm{ng} / \mathrm{ml}$ definition (the Phoenix definition) [11]. Toxicity was defined as any symptom developing after implantation and scored by the Common Terminology Criteria for Adverse Events (CTCAE), version 4.0. Urinary morbidity was assessed using the International Prostate Symptom Score (IPSS) before treatment and at 3 months, 6 months, and yearly after implantation. Sexual function was assessed using International Index of Erectile Function (IIEF-15). In addition, patients were asked whether or not they had erectile dysfunction, which was defined as the inability to achieve and/or maintain an erection sufficient for satisfactory sexual performance.

\section{Statistics}

Kaplan-Meier curves for FBF were generated for all patients based on risk category. Univariate analysis was calculated using a log-rank test to evaluate the association of risk factors with FBF. Statistical significance was defined as $p<0.05$. The sample size was too small to generate a reliable multivariate analysis.

\section{Results}

The median age of the cohort at the time of seed implantation was 64.0 years (range $46-78$ years). The median initial prostate-specific antigen level was $13 \mathrm{ng} / \mathrm{ml}$ (range 2.1 to 133 ), and the combined Gleason score was 2-6 in $57(52.3 \%), 7$ in $26(28.8 \%)$, and $8-10$ in $7(7.7 \%)$ of the 90 study subjects. The clinical stage distribution was T1 in $28(31.1 \%), \mathrm{T} 2$ in $57(51.3 \%)$, and T3 in $7(7.7 \%)$ of the patients (Table 1 ).

\section{PSA control}

After a median follow-up of 58 months (range 19-84), biochemical (PSA) failure occurred in 12 (13.3\%) patients. The median time to biochemical failure was 36 months (range 14-46 months). There were 4 deaths $(6.4 \%)$, of which one was prostate cancer related. FBF rates for clinical stage $\mathrm{T} 1, \mathrm{~T} 2$, and $\mathrm{T} 3$ were $96.4 \%, 85.9 \%$, and $60 \%$, respectively. With regards to NCCN risk assessment, FBF for low, intermediate, and high-risk cases were $95.4 \%$, $90.9 \%$, and $70.8 \%$, respectively.

Univariate analysis was performed to evaluate the association of clinical $\mathrm{T}$ stage, Gleason score (2 to 6, or 7 or 8 to 6), PSA group (less than or equal to $10 \mathrm{ng} / \mathrm{ml}$, more than 10 to $20 \mathrm{ng} / \mathrm{ml}$, and greater than $20 \mathrm{ng} / \mathrm{ml}$ ), and NCCN risk assessment with FBF. Clinical T stage $(p=0.041)$, Gleason score $(p=0.0038)$, and NCCN risk assessment $(p=0.057)$ were prognostic for biochemical control. Pre-treatment PSA was an insignificant factor in predicting FBF $(p=0.6)$. These results are illustrated in Figure 2.

\section{Morbidity}

Urinary morbidity was common but minor. Sixty-nine patients $(76.6 \%)$ experienced grade 1 and 2 urinary symptoms in the form of frequency, urgency, and poor stream. These symptoms were mostly self-limiting and occurred during the first two months after seed implantation. The IPSS score increased in the first two months then decreased during the sixth month. Acute urinary retention (grade 2) occurred in 4 patients $(4.4 \%$ ) within a month after implantation. Two of these patients voided successfully after 2 weeks of catheterization. However, the other two failed to void without catheterization, which necessitated

Table 1. Patient, cancer, treatment parameters, and freedom from biochemical failure (FBF)

\begin{tabular}{|c|c|c|}
\hline $\begin{array}{l}\text { Patient/tumour/ } \\
\text { treatment characteristics }\end{array}$ & $N=90$ & $\begin{array}{c}n=12 \\
\text { (biochemical recurrence) } \\
(\% \text { FBF })\end{array}$ \\
\hline \multicolumn{3}{|l|}{ Age (years) } \\
\hline Median & 64 & \\
\hline Mean & 63 & \\
\hline Range & $46-78$ & \\
\hline \multicolumn{3}{|l|}{ T stage } \\
\hline $\mathrm{T} 1$ & 28 & $1(96.4 \%)$ \\
\hline T2 & 57 & $8(85.9 \%)$ \\
\hline T3 & 5 & $3(60 \%)$ \\
\hline \multicolumn{3}{|l|}{ Gleason score } \\
\hline $2-6$ & 57 & $3(94.7 \%)$ \\
\hline 7 & 26 & $5(80.7 \%)$ \\
\hline $8-10$ & 7 & $4(57.1 \%)$ \\
\hline \multicolumn{3}{|l|}{ Pre-treatment PSA (ng/ml) } \\
\hline Median & 13 & \\
\hline Mean & 19 & \\
\hline Range & $2.1-133$ & \\
\hline$\leq 10$ & 26 & $2(92 \%)$ \\
\hline$>10-20$ & 44 & $5(88.6 \%)$ \\
\hline$>20$ & 20 & $5(70.0 \%)$ \\
\hline \multicolumn{3}{|l|}{ NCCN risk assessment } \\
\hline Low & 22 & $1(95.4 \%)$ \\
\hline Intermediate & 44 & $4(90.9 \%)$ \\
\hline High & 24 & $7(70.8 \%)$ \\
\hline \multicolumn{3}{|l|}{ Type of implant } \\
\hline Radical & 57 & \\
\hline Boost & 33 & \\
\hline
\end{tabular}

PSA - prostate specific antigen, NCCN - National Comprehensive Cancer Network 
A

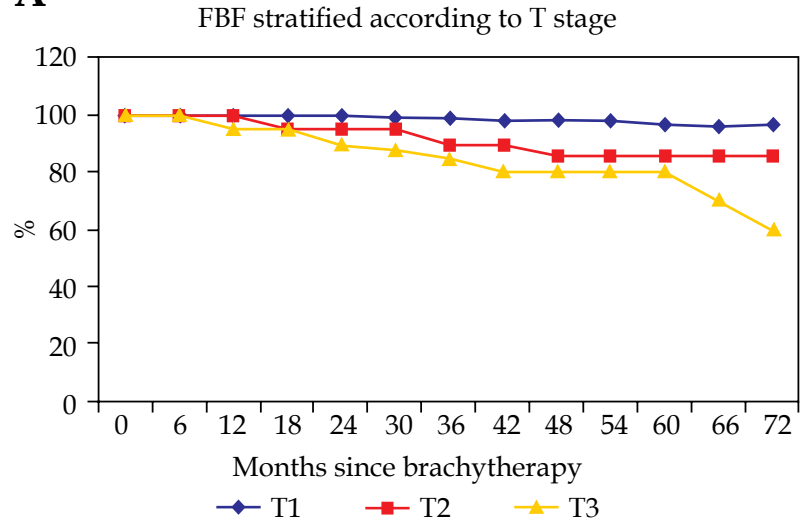

C

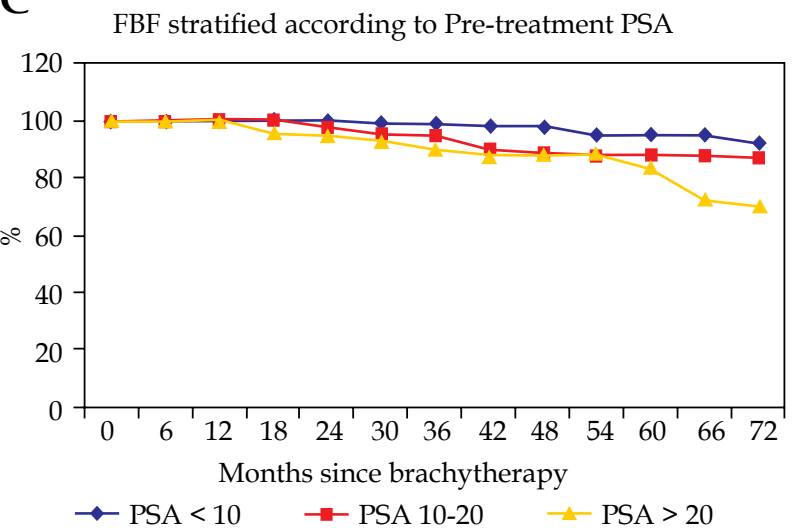

B

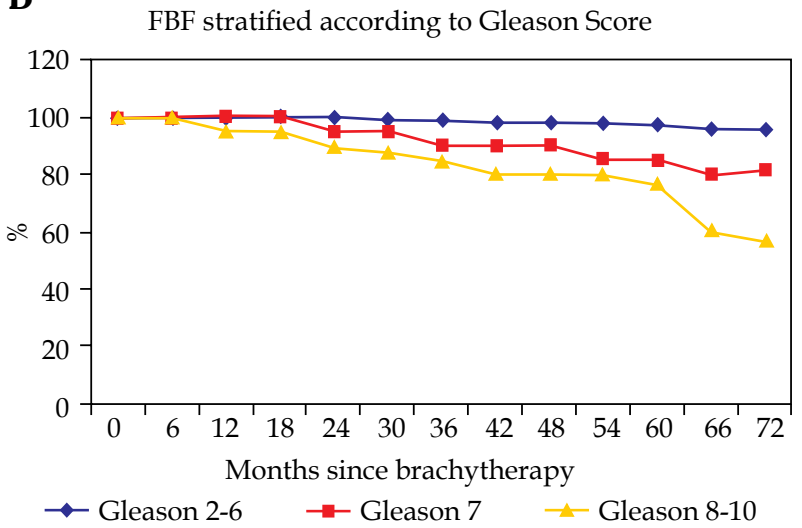

D

FBF stratified according to NCCN Risk Assessment

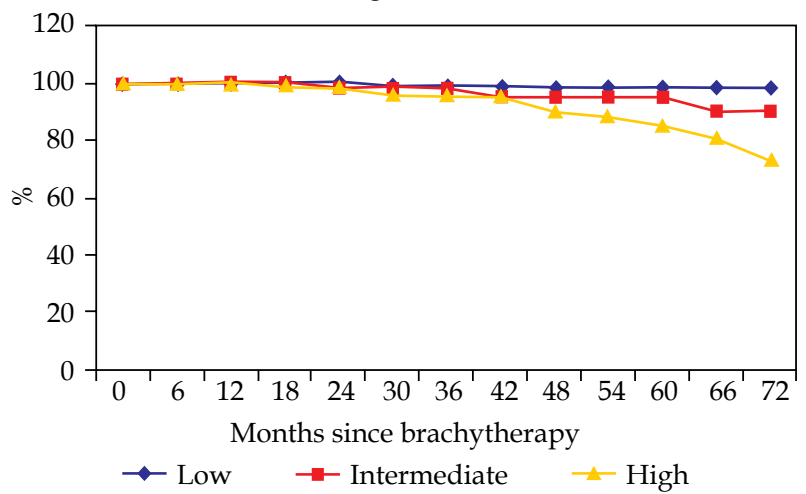

Fig. 2. Freedom from biochemical failure (FBF) stratified by risk category (A) T stage, (B) Gleason score, (C) pre-treatment PSA, (D) NCCN Risk Assessment

the insertion of a suprapubic catheter and transurethral resection of the prostate (grade 3) 9 months after seed insertion. They are currently fully continent and voiding well.

Gastrointestinal (GI) toxicity in the form of grade 2 rectal bleeding occurred in 3 patients. One patient developed a recto urethral fistula (grade 3 ) following banding of suspected hemorrhoids by a general surgeon 18 months after seed implantation. All four patients received a combination of brachytherapy and EBRT. None of the patients treated with seed implants alone experienced any GIT symptoms.

Normal erectile function was reported in 74 men $(82 \%)$ prior to treatment. In patients with over 2 years' follow-up, $62 \%$ reported good erection without the use of a phosphodiesterase 5 inhibitor (PD5I), 30\% require phosphodiesterase 5 inhibitor for good erection, and $8 \%$ had no erection.

\section{Discussion}

Prostate cancer is the most commonly diagnosed malignancy in men and the second most common cause of male cancer death after liver cancer in Ghana [12]. Ethnicity has a great influence on prostate cancer incidence and mortality rates, with the Black race having a two-fold higher prostate cancer death rate than the White race [13], which in turn has a two-fold higher incidence of prostate cancer death rate than the Asian race. The incidence of prostate cancer has risen substantially in the past decade partly due to the introduction of PSA based screening programs [14]. As prostate cancer awareness increases, so does it screening, which in particular results in an increase of early-stage or localized disease $[15,16]$. For localized prostate cancer, different curative treatment options are available. These include radical prostatectomy, external beam radiotherapy, and prostate brachytherapy as low or high dose rate procedure. Low dose rate permanent brachytherapy currently involves the use of ${ }^{125} \mathrm{I}$ and ${ }^{103} \mathrm{Pd}$ radioisotopes, which deliver the intended dose over a period of time depending on the dose rate of the isotope. High dose rate brachytherapy, on the other hand, involves the temporary use of ${ }^{192} \mathrm{Ir}$, an isotope that is able to deliver the intended dose over a shorter period of time [17]. To date, no significant differences in outcome have been shown between these three treatment options in early stage disease $[18,19]$. High intermediate and high risk patients require a combination of hormone deprivation therapy, and external beam with or without brachytherapy [19]. Besides tumor control, treatment morbidity is also an important endpoint that should be taken into account in the assessment of any treatment option.

\section{Prostate specific antigen control}

It is now generally accepted that post-treatment PSA levels are the most sensitive means of assessing disease 
control and determining the efficacy of a particular treatment [20]. Increasing prostate specific antigen (PSA) levels after curative treatment is the first indicator of disease recurrence. Biochemical failure is often used as a reliable marker for treatment efficacy and prostate cancer survival. Several studies have shown that the primary pre-treatment factors associated with PSA control are pre-treatment PSA, the Gleason score, and the clinical stage $[4,5,21]$. This makes PSA control comparisons across single-institution series problematic because case selection will have a significant impact on the outcome of treatment. The proportion of high risk parameters in our study was much higher than that reported in most brachytherapy series. This is consistent with a report by Yamoah et al. [22], on a total of 2268 patients, which included 81\% Caucasians, 12\% African Americans (AA) $6 \%$ Hispanics, and $1 \%$ Asians. The 10 -year actuarial FBF rate was $70 \%$ for AA men and $84 \%$ for all others $(p=0.002)$. Similar to our findings, a greater percentage of AA men presented with higher prostate-specific antigen levels (PSA) (> $10 \mathrm{ng} / \mathrm{ml} ; 44 \%$ vs. $21 \% ; p<0.001)$ and had higher risk disease $(24 \%$ vs. $15 \% ; p<0.001)$ compared with Caucasian men. Despite these racial differences in pre-treatment parameters and biochemical disease recurrence, our results compare favorably with those reported by other institutions (Table 2) with greater brachytherapy experience.

We think that two reasons might have contributed to the favorable results in our first cohort of patients. First, the equipment involved in the LDR brachytherapy procedure (ultrasonography, perineal template, stepping unit, prostate needles, and treatment planning system) improves the precision of the implant. Secondly, we believe that the teaching, training and proctoring that our brachytherapy team received was of high quality.

\section{Side effects}

Tumor control and survival rates for low-risk prostate cancer are comparable between the different treatment modalities. Therefore, additional outcome measures and patient preferences should be evaluated prior to treatment selection. Toxicity and quality of life have become important endpoints considered by most patients in the process of selecting a treatment modality $[25,26,27]$. Recent reports show that among the primary treatments for prostate cancer (brachytherapy, radical prostatectomy, and external beam radiation therapy - EBRT), brachyther- apy has the least effect on over-all quality of life [28]. The biggest negative effect of brachytherapy is related to irritative and obstructive urinary symptoms. Our experience with these initial patients shows that most patients experience some degree of deterioration in lower urinary tract symptoms that may last for a few weeks to months. This is a consequence of the large dose delivered to the prostate in the first month, and the edema and hematoma caused by the trauma of the needles [29]. The symptoms are generally mild and of short duration, but may be a bother to patients who expect rapid recovery and return to pre-implant health. The predominant acute toxicity after prostate brachytherapy is urinary retention requiring catheterization, which occurred in $4 \%$ of our patients. Published acute urinary retention (AUR) rates vary from $1 \%$ to $34 \%[30,31]$. Patients pre-existing urinary obstructive symptoms are particularly prone to development of acute retention.

Rectal toxicity after prostate brachytherapy develops more often as a late side effect, and occurs approximately one year after the procedure and persists for 1 to 2 years. Grade 2 and 3 rectal toxicities have been reported to occur in 2 to $12 \%$ and 0.4 to $2 \%$ of patients, respectively [32,33]. Additionally, patients who receive supplemental external beam boost are prone to develop rectal toxicity as a result of telangiectasia $[34,35]$. This is consistent with our observation that all three patients who developed grade 2 rectal bleeding had received supplemental EBRT.

Recto urethral fistula, a very distressing but rare toxicity, was observed in one patient $(1 \%)$. The reported incidence of this complication is less than $0.1 \%[36,37]$, and most cases occurred following rectal instrumentation or rectal biopsies after seed implantation [38] as occurred in our patient. This can be mitigated with the education of patients and rectal surgeons. The single patient who died of prostate cancer 4 years after seed implantation was probably under staged.

One of the most prominent advantages of brachytherapy is potency preservation. Very large series have reported a 5-year potency rates after prostatectomy, EBRT, and brachytherapy of $40 \%, 75 \%$, and $80 \%$, respectively [39], suggesting that brachytherapy may be the best option for prostate cancer treatment from a sexual function quality-of-life viewpoint. Our characterization of sexual function with the International Index of Erectile Function (IIEF 15) demonstrated that $62 \%$ patients who had undergone brachytherapy were able to preserve their potency.

Table 2. Freedom from biochemical failure (FBF) of ${ }^{125}$ l low-dose-rate brachytherapy according to NCCN risk assessment

\begin{tabular}{lcccc} 
Reference series & Number of patients & Follow-up (years) & Low risk & Intermediate risk \\
\hline Stone and Stock 2014 [1] & 1669 & 10 & 96 & 97.5 \\
\hline Yorozu et al. 2014 [23] & 1313 & 7 & 98 & 93.2 \\
\hline Marshall et al. 2014 [4] & 2495 & 12 & 90 & 81 \\
\hline Guedea et al. 2006 [24] & 1050 & 4 & 91 & 93 \\
\hline This study & 90 & 7 & 95.4 & 98.9
\end{tabular}




\section{Conclusions}

Our results strongly support brachytherapy as a highly effective treatment option for low, intermediate, and selected high-risk prostate cancer. Urinary, rectal, and sexual side effects were tolerable. Our initial experience provides a potential road map for the transfer of technology from developed countries to developing ones as it is difficult for practitioners from sub-Saharan Africa to acquire hands-on skill in complex surgical procedures in advanced countries. Training in the practitioner's country with proctoring from experts is the ideal platform for rapid transfer of knowledge and skills. We expect that with the local availability of this treatment modality many more West African men with localized prostate cancer will undergo brachytherapy in the near future; additionally, the local center may provide training opportunities for urologist and radiation oncologist in the sub region. Limitations of this study include small patient numbers and short follow-up period.

\section{Disclosure}

Authors report no conflict of interest.

\section{References}

1. Stone NN, Stock RG. 15-year cause specific and all-cause survival following brachytherapy for prostate cancer: negative impact of long-term hormonal therapy. J Urol 2014; 192: 754-759.

2. Arvold ND, Chen MH, Moul JW et al. Risk of death from prostate cancer after radical prostatectomy or brachytherapy in men with low or intermediate risk disease. J Urol 2011; 186: 91-96.

3. Walsh PC, DeWeese TL, Eisenberger MA. Clinical practice. Localized prostate cancer. N Engl J Med 2007; 357: 2696-2705.

4. Marshall RA, Buckstein M, Stone NN et al. Treatment outcomes and morbidity following definitive brachytherapy with or without external beam radiation for the treatment of localized prostate cancer: 20-year experience at Mount Sinai Medical Center. Urol Oncol 2014; 32: 381-387.

5. Guinot JL, Ricós JV, Tortajada MI et al. Comparison of permanent 125 I seeds implants with two different techniques in 500 cases of prostate cancer. J Contemp Brachytherapy 2015; 4: 258-264.

6. Aronowitz JN, Rivard MJ. The phylogeny of permanent prostate brachytherapy. J Contemp Brachytherapy 2013; 5: 89-92.

7. Rebbeck TR, Zeigler-Johnson CM, Heyns CF et al. Prostate cancer screening, detection and treatment practices, among Sub-Saharan African urologists. African J Urol 2011; 17: 85-91.

8. Egner JR. AJCC Cancer Staging Manual. JAMA 2010; 304: 1726.

9. Mohler JL, Armstrong AJ, Bahnson RR et al. Prostate cancer, Version 3.2012: featured updates to the NCCN guidelines. J Natl Compr Cancer Netw 2012; 10: 1081-1087.

10. Potters L, Calguaru E, Thornton KB et al. Toward a dynamic real-time intraoperative permanent prostate brachytherapy methodology. Brachytherapy 2003; 2: 172-180.

11. Roach M 3rd, Hanks G, Thames H et al. Defining biochemical failure following radiotherapy with or without hormonal therapy in men with clinically localized prostate cancer: Recommendations of the RTOG-ASTRO Phoenix Consensus Conference. Int J Radiat Oncol Biol Phys 2006; 65: 965-974.

12. Wiredu EK, Armah HB. Cancer mortality patterns in Ghana: a 10 -year review of autopsies and hospital mortality. BMC Public Health 2006; 6: 159.
13. American Cancer Society. Cancer Facts \& Figures for African Americans 2013-2014. 2013. http://www.cancer.org/acs/ groups/content/@epidemiologysurveilance/documents/ document/acspc-036921.pdf

14. Howrey BT, Kuo Y-F, Lin Y-L et al. The impact of PSA screening on prostate cancer mortality and overdiagnosis of prostate cancer in the United States. J Gerontol A Biol Sci Med Sci 2013; 68: $56-61$.

15. Finne P, Fallah M, Hakama M et al. Lead-time in the European randomized study of screening for prostate cancer. Eur J Cancer 2010; 46: 3102-3108.

16. Hsing AW, Yeboah E, Biritwum R et al. High prevalence of screen-detected prostate cancer in West Africans: implications for racial disparity of prostate cancer. J Urol 2014; 192: 730-736.

17. Wojcieszek P, Białas B. Prostate cancer brachytherapy: guidelines overview. J Contemp Brachytherapy 2012; 4: 116-120.

18. Fisher CM, Troncoso P, Swanson D et al. Knife or needles? A cohort analysis of outcomes after radical prostatectomy or brachytherapy for men with low- or intermediate-risk adenocarcinoma of the prostate. Brachytherapy 2014; 11: 429-434.

19. Heidenreich A, Bellmunt J, Bolla M et al. EAU guidelines on prostate cancer. Part 1: screening, diagnosis, and treatment of clinically localized disease. Eur Urol 2011; 59: 61-71.

20. Wallace TJ, Torre T, Grob M et al. Current Approaches, Challenges and Future Directions for Monitoring Treatment Response in Prostate Cancer. J Cancer 2014; 5: 3-24.

21. Nobes JP, Wells IG, Khaksar SJ. Biochemical relapse-free survival in 400 patients treated with I-125 prostate brachytherapy: The Guildford experience. Prostate Cancer Prostatic Dis 2009; 12: 61-66.

22. Yamoah K, Stone N, Stock R. Impact of race on biochemical disease recurrence after prostate brachytherapy. Cancer 2011; 117: 5589-5600

23. Yorozu A, Kuroiwa N, Takahashi A et al. Permanent prostate brachytherapy with or without supplemental external beam radiotherapy as practiced in Japan: Outcomes of 1300 patients. Brachytherapy 2014; 14: 111-117.

24. Guedea F, Aguilo F, Polo A et al. Early biochemical outcomes following permanent interstitial brachytherapy as monotherapy in 1050 patients with clinical T1-T2 prostate cancer. Radiother Oncol 2006; 80: 57-61.

25. Anandadas CN, Clarke NW, Davidson SE et al. Early prostate cancer - which treatment do men prefer and why? BJU Int 2011; 107: 1762-1768.

26. Mahal BA, Chen MH, Bennett CL. The association between race and treatment regret among men with recurrent prostate cancer. Prostate Cancer Prostatic Dis 2015; 18: 38-42.

27. Acar C, Schoffelmeer CC, Tillier C et al. Quality of life in patients with low-risk prostate cancer. A comparative retrospective study: brachytherapy versus robot-assisted laparoscopic prostatectomy versus active surveillance. J Endourol 2014; 28: 117-124.

28. Chira C, Delouya G, Larrivée $S$ et al. Prostate volume changes during permanent seed brachytherapy: an analysis of intra-operative variations, predictive factors and clinical implication. Radiat Oncol 2013; 8: 177-180.

29. Stone NN. Transurethral resection versus intermittent catheterization in patients with retention after combined brachytherapy/external beam radiotherapy for prostate cancer. J Urol 2013; 189: 800-801.

30. Elshaikh MA, Angermeier K, Ulchaker JC et al. Effect of anatomic, procedural, and dosimetric variables on urinary retention after permanent iodine-125 prostate brachytherapy. Urology 2003; 61: 152-155.

31. Talcott JA, Clark JA, Stark PC et al. Long-term treatment related complications of brachytherapy for early prostate can- 
cer: a survey of patients previously treated. J Urol 2001; 166: 494-499.

32. Sarosdy MF. Urinary and rectal complications of contemporary permanent transperineal brachytherapy for prostate carcinoma with or without external beam radiation therapy. Cancer 2004; 101: 754-760.

33. Tanaka N, Asakawa I, Anai S. Periodical assessment of genitourinary and gastrointestinal toxicity in patients who underwent prostate low-dose-rate brachytherapy. Radiat Oncol 2013; 8: 25.

34. Chen AB, D'Amico AV, Neville BA et al. Patient and treatment factors associated with complications after prostate brachytherapy. J Clin Oncol 2006; 24: 5298-5304.

35. Chrouser KL, Leibovich BC, Sweat SD et al. Urinary fistulas following external radiation or permanent brachytherapy for the treatment of prostate cancer. J Urol 2005; 173: 1953-1957.

36. Harada K, Ishikawa $\mathrm{H}$, Saito $\mathrm{Y}$ et al. Risk factors for rectal bleeding associated with I-125 brachytherapy for prostate cancer. J Radiat Res 2012; 53: 923-929.

37. Wallner K, Sutlief S, Bergsagel C. Severe rectal complications after prostate brachytherapy. Radiother Oncol 2015; 114: 272-275.

38. Pardo Y, Guedea F, Aguiló F et al. Quality-of-life impact of primary treatments for localized prostate cancer in patients without hormonal treatment. J Clin Oncol 2010; 28: 4687-4696.

39. Buron C, Le Vu B, Cosset JM et al. Brachytherapy versus prostatectomy in localized prostate cancer: results of a French multicenter prospective medico-economic study. Int J Radiat Oncol Biol Phys 2007; 67: 812-822. 